\title{
A USEFUL FUNCTOR AND THREE FAMOUS EXAMPLES IN TOPOLOGY(1)
}

\author{
BY \\ R. F. WILLIAMS
}

The purpose of this note is to describe a functor which provides a framework for certain constructions in topology. It is related to the sets $(E, \pi, B, X, q)$ described in [7] and is particularly adapted to discussing the limit of repeated modifications of triangulable spaces. Roughly speaking, one forms a space $X \Delta K$ by replacing each top dimensional simplex of a complex $K$ with a copy of a space $X$. If in addition there are mappings on the spaces $X, K$, these induce a mapping on the new space $X \Delta K$.

It has been called to my attention that several authors have considered analogous functors (though not as far as I know, in written form). This is not surprising inasmuch as $X \Delta K$ is defined just as the Whitney sum of two bundles.

Though the principal applications of this functor are to be found elsewhere, in a paper by Frank Raymond and the author [8; 7] and a forthcoming paper by the author, three famous examples, Pontrjagin [6], Boltyanskii [2], and Kolmogoroff [5] are given as applications in the last section. Two of these are in dimension theory proper, but the third is essentially about transformation groups. This was pointed out by Professor Deane Montgomery whom the author would like to thank for his considerable aid and encouragement.

It is hoped that the reader will find our description of Boltyanskii's example easier than the original, as a simpler, more homogeneous version is given. In addition, in our version of Kolmogoroff's example, the group acts without fixed points. This answers a question raised by Anderson [1].

1. Definitions. Throughout this section $n$ is a fixed integer $\geqq 0$ and $s$ is the standard $n$-simplex with vertices $v_{0}, v_{1}, \cdots, v_{n}$. $s$ will be regarded as a complex and as being closed when thought of as a space. Otherwise all simplexes, $\sigma, \tau, \rho$ are taken as open and $\sigma \in K$ means $\sigma$ is a simplex of $K$. The dimension of a complex $K$ is the maximum of the dimensions of the simplexes of $K$.

For an $n$-complex $K$ there is a natural map $\phi_{K}: K^{\prime} \rightarrow s$, where $K^{\prime}$ is the barycentric subdivision of $K$ and $\phi_{K}$ sends $b\left(\sigma^{i}\right)$ (the barycenter of an $i$-simplex of $K)$ into the $i$ th vertex, $v_{i} \in s$. This extends uniquely to the simplicial map $\phi_{K}$.

Received by the editors February 15, 1962.

(1) National Science Foundation Fellow, 1959-1961 This research was supported in part by contract No. NSF G-10369. 
Note that $\phi_{K} \mid \bar{\sigma}$ is a homeomorphism for each $\sigma \in K$. Also that if $L$ is another $n$-complex and $t: K \rightarrow L$ is a map which is

(M2) simplicial and collapses no simplex then

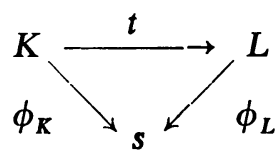

is commutative.

Now suppose

(01) $X$ is a topological space and $g: X \rightarrow s$.

Define $X \Delta_{q} K=\left\{(x, k) \in X \times K: q x=\phi_{K} k\right\}$. This will usually be written simply $X \Delta K$. Then for $t: K \rightarrow L$ satisfying (M2) we define

$$
1 \Delta t: X \Delta K \rightarrow X \Delta L
$$

by $(1 \Delta t)(x, k)=(x, t k)$. Note that $(1 \Delta u)(1 \Delta t)=1 \Delta u t$, where defined.

The identity map $1: s \rightarrow s$ gives $s \Delta K \approx K^{\prime}$ and we will identify these spaces via the map $(x, k) \rightarrow k$. Now consider the diagram

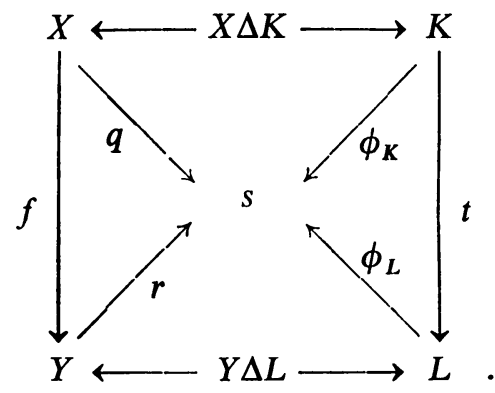

If the triangle on the left is commutative then there will clearly be an induced map $f \Delta t: X \Delta K \rightarrow Y \Delta L$. But this is too great a restriction upon the map $f$ and we require only that

(M1) $r f q^{-1}(\sigma) \subset \bar{\sigma}$, for each simplex $\sigma \in s$.

Given this, define

by

$$
f \Delta 1: X \Delta K \rightarrow Y \Delta K
$$

$$
(f \Delta 1)(x, k)=\left(f x,(\phi \mid \bar{\sigma})^{-1} r f x\right), \quad \text { where } \sigma \in K^{\prime}, k \in \bar{\sigma} .
$$

There are four possible difficulties with this definition. In order that $f \Delta 1$ be

(1) defined at all, we need to know that $r f x \in \phi(\bar{\sigma})=$ domain of $(\phi \mid \bar{\sigma})^{-1}$;

(2) well defined, we need $k \in \bar{\sigma}, \bar{\tau}$ to imply $(\phi \mid \bar{\sigma})^{-1} r f x=(\phi \mid \bar{\tau})^{-1} r f x$;

(3) continuous, we need only check (1) and (2);

(4) a map into $Y \Delta K$, we need only note that $r(f x)=\phi(\phi \mid \bar{\sigma})^{-1} r f x$.

Thus we check $(1,2)$ : first $q x=\phi k \in \phi(\bar{\sigma})$ so that $x \in q^{-1} \phi(\bar{\sigma})$ and hence $r f x$ $\in r f q^{-1}(\phi \bar{\sigma}) \subset \phi \bar{\sigma}$. Secondly, suppose $k \in \bar{\sigma}, \bar{\tau}$ and $k \in \rho$, say. Then $\rho$ is a face of both $\sigma$ and $\tau$. Hence 


$$
(\phi \mid \bar{\sigma})^{-1}\left|\phi(\bar{\rho})=(\phi \mid \bar{\rho})^{-1}=(\phi \mid \bar{\tau})^{-1}\right| \phi(\bar{\rho}),
$$

so that $(\phi \mid \bar{\sigma})^{-1} r f x=(\phi \mid \bar{\tau})^{-1} r f x$.

REMARK. $X \Delta K$ may be interpreted as follows. Let $X_{\sigma}=\{(x, k) \in X \Delta K: k \in \bar{\sigma}\}$, for $\sigma \in K^{\prime}$. For an $n$-simplex $\sigma$, the map $X \rightarrow X_{\sigma}$ which sends $x \rightarrow\left(x,(\phi \mid \bar{\sigma})^{-1} q x\right)$ is a homeomorphism. Similarly for $\rho$ an $i$-simplex in $K^{\prime}, X_{\rho} \approx q^{-1}(\phi \bar{\rho})=q^{-1}\left(\bar{\rho}^{\prime}\right)$ and $\rho^{\prime}$ is an $i$-simplex of $s$. Hence for two $n$-simplexes $\sigma, \tau \in K^{\prime}, \rho$ their common face, then $X_{\sigma}$ and $X_{\tau}$ are copies of $X$ identified along their respective copies of $X_{\rho}$.

Among the immediate consequences of the definitions are

(1.1) $(1 \Delta u)(1 \Delta t)=1 \Delta u t$, where $u, t$ satisfy (M2).

(1.2) $(g \Delta 1)(f \Delta 1)=g f \Delta 1$, where the following triangles satisfy (M1),

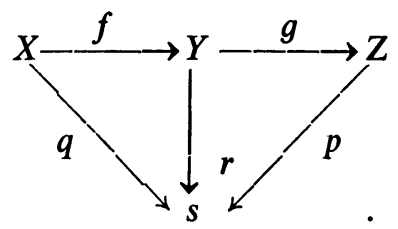

(1.3) the following diagram is commutative:

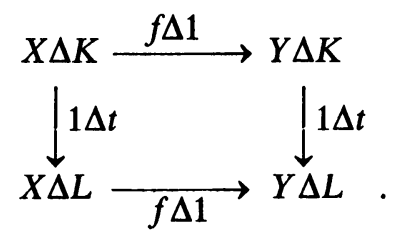

We will verify (1.3); going over and down:

$$
(x, k) \rightarrow\left(f x,(\phi \mid \bar{\sigma})^{-1} r f x\right) \rightarrow\left(f x, t(\phi \mid \bar{\sigma})^{-1} r f x\right) ;
$$

down and over:

$$
(x, k) \rightarrow(x, t k) \rightarrow\left(f x,(\theta \mid t \bar{\sigma})^{-1} r f x\right),
$$

where $\phi=\phi_{K}$ and $\theta=\phi_{L}$ are the maps described above. Then these two maps agree as all maps in the following commutative triangle are 1-1:

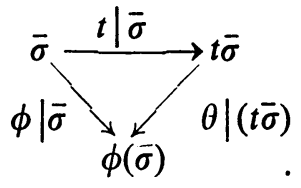

Then in general define $f \Delta t=(f \Delta 1)(1 \Delta t)=(1 \Delta t)(f \Delta 1)$.

(1.4) $q \Delta 1: X \Delta K \rightarrow K^{\prime}$ is defined by $(q \Delta 1): X \Delta K \rightarrow s \Delta K \approx K^{\prime}$ by our identification above. Note $q \Delta 1$ is the restriction of the projection $X \times K \rightarrow K$ to $X \Delta K$. 
2. Simplicial theory and chain maps. An additional hypothesis is needed to insure that $X \Delta K$ be a complex. One that suffices is

(S01) $X$ is a complex, $q^{-1}(\bar{\sigma})$ is a subcomplex for all $\sigma \in s$, and

(SM1) $f: X \rightarrow Y$ is simplicial and satisfies (M1).

REMARK. One may always assume that $q: X \rightarrow s$ is simplicial relative to some subdivision of $s$. For given $X, q$ satisfying (S01), there is a simplicial approximation $q^{\prime}$ (relative to some subdivision of $s$ ) to $q$ such that $q^{\prime} q^{-1}(\sigma) \subset \sigma$ for all $\sigma \in s$. Such a $q^{\prime}$ exists by induction on the skeleta $s_{0}, s_{1}, \cdots, s_{n}$ of $s$.

Then $q^{-1}(\sigma)=q^{\prime-1}(\sigma)$ for all $\sigma \in s$ and hence there are the maps

$$
1 \Delta 1: X \Delta_{q} K \rightarrow X \Delta_{q^{\prime}} K, \quad 1 \Delta 1: X \Delta_{q}, K \rightarrow X \Delta_{q} K
$$

and their composition in either order is the identity. Also if $f: X \rightarrow Y, r: Y \rightarrow s$, satisfy (SM1) then $r^{\prime} f q^{\prime-1}(\bar{\sigma})=r f q^{-1}(\bar{\sigma}) \subset \bar{\sigma}$, for all $\sigma \in s$ so that the replacement $q \rightarrow q^{\prime}$ is natural.

However the weaker requirement (S01) seems more "natural" and will be used. We remain in this simplicial category in all that follows.

(2.1) Lemma. If $q: X \rightarrow s$ satisfies (S01) then $X \Delta K$ has a natural simplicial structure.

Proof. The proof is in four parts. In $(1,2)$ we triangulate $X \Delta K$ and in $(3,4)$ show that the induced maps are simplicial.

(1) For $\sigma \in K^{\prime}$ define $X_{\sigma}=\{(x, k) \in X \Delta K: k \in \bar{\sigma}\}$. Then $h_{\sigma}(x)=\left(x,(\phi \mid \bar{\sigma})^{-1} q x\right)$ defines a homeomorphism $h_{\sigma}: q^{-1} \phi \bar{\sigma} \rightarrow X_{\sigma} . h_{\sigma}$ is clearly 1-1 and if $(x, k) \in X_{\sigma}$, then $q x=\phi k \in \phi \bar{\sigma}$ so that $h_{\sigma}(x)=\left(x,(\phi \mid \bar{\sigma})^{-1} q x\right)=\left(x,(\phi \mid \bar{\sigma})^{-1} \phi k\right)=(x, k)$. But by hypothesis $q^{-1} \phi \bar{\sigma}$ is a complex so that $h_{\sigma}$ triangulates $X_{\sigma}$.

(2) If $\tau$ is a face of $\sigma \in K^{\prime}$, then $\left(h_{\sigma} \mid q^{-1} \phi \bar{\tau}\right) x=\left(x,(\phi \mid \bar{\tau})^{-1} q x\right)=\left(x,(\phi \mid \bar{\tau})^{-1} q x\right)$ $=h_{\tau} x$, for all $x \in q^{-1} \phi(\bar{\tau})$. Thus the triangulations $h_{\sigma}$ defined in part (1) agree where they intersect and taken together triangulate $X \Delta K$. If $X$ and $K$ are finite complexes so is $X \Delta K$.

(3) Now suppose in addition that $r: Y \rightarrow s$ satisfies (S01) and $f: X \rightarrow Y$ satisfies (SM1). In order to show that $f \Delta 1: X \Delta K \rightarrow Y \Delta K$ is simplicial suppose $\rho \in X \Delta K$. Then $\rho$ is a simplex of $X_{\sigma}$ for some $\sigma \in K^{\prime}$. That is, there is a simplex $\rho_{1} \in q^{-1} \phi \bar{\sigma} \subset X$ such that $h_{\sigma}\left(\rho_{1}\right)=\rho$. Then $\rho_{2}=f \rho_{1}$ is a simplex in $r^{-1} \phi \bar{\sigma}$ because $r f q^{-1}(\bar{\sigma}) \subset \bar{\sigma}$. Now by definition $(f \Delta 1)\left(\rho_{1}\right)=\left(f \rho_{1},(\phi \mid \bar{\sigma})^{-1} r f \rho_{1}\right)=\left(\rho_{1},(\phi \mid \bar{\sigma})^{-1} r \rho_{2}\right)$ and this last is a simplex in the natural triangulation of $Y \Delta K$.

(4) Varying the second factor suppose $t: K \rightarrow L$ satisfies (M1). Then $(1 \Delta t)\left(\rho,\left(\phi_{K} \mid \bar{\sigma}\right)^{-1} q \rho\right)=\left(\rho, t\left(\phi_{K} \mid \bar{\sigma}\right)^{-1} q \rho\right)=\left(\rho,\left(\phi_{L} \mid t \bar{\sigma}\right)^{-1} q \rho\right)$ which is a simplex of $X \Delta L$.

(2.2) Notation. A point $(x, k) \in X \Delta K \subset X \times K$ will be denoted at times by by $x \Delta k$. A simplex $\tau \in X_{\sigma} \subset X \Delta K$ has the form $\tau=h_{\sigma}(\rho)=\left(\rho,(\phi \mid \bar{\sigma})^{-1} q \rho\right)$ where $\sigma \in K^{\prime}$. We introduce the notation $(\rho, \sigma)=\tau$; note $(\rho, \sigma) \neq \rho \Delta \sigma$ as this last in- 
volves a number of copies of $\rho$ depending upon the dimensions of $\rho$ and $\sigma$. The notation $(\rho, \sigma)$ is far from unique, for if $\sigma$ is a face of $\sigma^{\prime}$, then $(\rho, \sigma)=\left(\rho, \sigma^{\prime}\right)$.

If $C \in C_{*}\left(q^{-1} \phi(\bar{\sigma}), A\right)$, i.e., $C=\sum \alpha_{i} \cdot \rho_{i}$ where $\rho_{i} \in q^{-1} \phi(\bar{\sigma})$, we let $(c, \sigma)$ denote $\sum \alpha_{i}\left(\rho_{i}, \sigma\right)$. One easily verifies the fact that $\partial(c, \sigma)=(\partial c, \sigma)$. Also one can check the formula $(q \Delta 1)_{\#}(c, \sigma)=\left(q_{\#} c, \sigma\right)=(\phi \mid \bar{\sigma})^{-1} q_{*} c$. The last $=$ follows from our identification $s \Delta K=K^{\prime}$.

Chain maPs. $\quad C_{*}(K, A) \rightarrow C_{*}(X \Delta K, A)$.

Now suppose $\gamma: C_{*}(s ; A) \rightarrow C_{*}(X ; A)$ is a chain map carried by $q^{-1}$. Then (2.3) $\gamma$ induces a chain map $\gamma \Delta 1: C_{*}(s \Delta K ; A) \rightarrow C_{*}(X \Delta K ; A)$ carried by $(q \Delta 1)^{-1}$. If $q_{*} \gamma=1$ then $(q \Delta 1)_{*}(\gamma \Delta 1)=1$.

Proof. Recall that $s \Delta K=K^{\prime}$. Then $\gamma \Delta 1$ is defined on elementary chains $\alpha \cdot \sigma$ of $C_{*}\left(K^{\prime}, A\right)$ by $(\gamma \Delta 1)(\alpha \cdot \sigma)=(\gamma(\alpha \cdot \phi \sigma), \sigma)$. Then clearly $\gamma \Delta 1$ is carried by $(q \Delta 1)^{-1}$. To check that $\gamma \Delta 1$ is a chain map:

If $q_{*} \gamma=1$, then

$$
\begin{aligned}
\partial((\gamma \Delta 1)(\alpha \cdot \sigma)) & =\partial(\gamma(\alpha \phi \sigma), \sigma)=(\partial \gamma(\alpha \cdot \phi \sigma), \sigma) \\
& =(\gamma(\alpha \cdot \partial \phi \sigma), \sigma)=(\gamma(\alpha \cdot \phi \partial \sigma), \sigma) \\
& =(\gamma \Delta 1)(\partial(\alpha \sigma)) .
\end{aligned}
$$

$$
\begin{aligned}
(q \Delta 1)_{*}(\gamma \Delta 1)(\alpha \sigma) & =(q \Delta 1)_{*}(\gamma(\alpha \cdot \phi \sigma), \sigma)=(\phi \mid \bar{\sigma})^{-1} q_{*} \gamma(\alpha \cdot \phi \sigma) \\
& =(\phi \mid \bar{\sigma})^{-1} 1(\alpha \cdot \phi \sigma)=\alpha \cdot \sigma .
\end{aligned}
$$

(2.4) Corollary. If there is a chain map $\gamma: C_{*}(s ; A) \rightarrow C_{*}(X ; A)$ such that $q_{*} \gamma=1$, then $(q \Delta 1)_{*}: H_{*}(X \Delta K ; A) \rightarrow H_{*}(K ; A)$ is onto.

Proof. For then the chain map $(\gamma \Delta 1): C_{*}(K ; A) \rightarrow C_{*}(X \Delta K ; A)$ induces a map $(\gamma \Delta 1)_{*}: H_{*}(K ; A) \rightarrow H_{*}(X \Delta K ; A)$, such that $(q \Delta 1)_{*}(\gamma \Delta 1)_{*}=1$. Hence $(q \Delta 1)_{*}$ is onto.

3. Applications. Throughout this section $s$ is the standard 2-simplex. We need the following results for compact metric spaces $X$. They can be found in Hurewicz-Wallman [4,p. 72] and [4, p. 152].

A. $\operatorname{dim} X \leqq n \Leftarrow$ for each $\varepsilon>0$ there exists an $\varepsilon$-map $f$ of $X$ into a complex $Y_{\varepsilon}$ of dimension $\leqq n$.

B. $\operatorname{dim} X \geqq n \Leftarrow H_{n}(X ; G) \neq 0$ for some coefficient group $G$. $(f: X \rightarrow Y$ is an $\varepsilon$-map if diameter $f^{-1}(y)<\varepsilon$ for all $y \in Y$.)

Now suppose $X$ is the inverse limit of a sequence

$$
X_{1} \stackrel{f_{1}}{\longleftarrow} X_{2} \stackrel{f_{2}}{\longleftarrow} X_{3} \longleftarrow \cdots
$$

where $X_{i}$ is a complex (simplicial or cellular) and $f_{i}$ is simplicial or cellular. Let $\sigma \in X_{i}$, set $X_{\sigma}=\left\{x \in X: x_{i} \in \sigma\right\}$, and define $d_{i}=\max _{\sigma \in K_{i}}$ (diameter $X_{\sigma}$ ). Assume that $d_{i} \rightarrow 0$ as $i \rightarrow \infty$. Then A, B can be reformulated as follows. 
$\mathrm{A}^{\prime} . \quad \operatorname{dim} X \leqq n \Leftarrow$ for each $i$ there is a simplicial (or cellular) approximation $g_{i}: X_{i+1} \rightarrow X_{i}$ to $f_{i}$ such that $g_{i}\left(X_{i+1}\right)_{i} \subset\left(n\right.$-skeleton of $\left.X_{i}\right)$.

B'. $\operatorname{dim} X \geqq n \Leftarrow$ for each $i, f_{i *}: H_{n}\left(X_{i+1} ; G\right) \rightarrow H_{n}\left(X_{i} ; G\right)$ is onto and nontrivial for at least one $i$.

For then the Čech homology $H_{n}(X ; G)$ is not zero.

I. (Pontruagin [6]). There exist compact metric spaces $\Phi_{p}$, one for each prime $p$, such that $\operatorname{dim} \Phi_{p}=2$ and

$$
\operatorname{dim} \Phi_{p} \times \Phi_{p^{\prime}}= \begin{cases}3, & \text { if } p \neq p^{\prime} \\ 4, & \text { if } p=p^{\prime}\end{cases}
$$

Proof. We consider only the case $p \neq p^{\prime}$. Let $X_{0}=S^{1} \times[0,1]$ and let $X_{p}$ be the identification space formed from $X_{0}$ by identifying $(\theta, 1) \sim(\theta+2 \pi / p, 1)$ for all $\theta \in S^{1}$. (Note: $X_{2}=$ Möbius band.) Let $B_{p}$ correspond to $S^{\prime} \times 0$ and $C_{p}$ to $S^{1} \times 1$. Then $\left(X_{p}, B_{p} \cup C_{p}\right)$ is a relative, orientable manifold and choosing orientations, $\partial X_{p}=B_{p}+p \cdot C_{p}$.

Define $q^{\prime}: X_{0} \rightarrow s$ by

$$
q^{\prime}: S^{1} \times[0,1] \rightarrow S^{1} \times[0,1 / 2] \rightarrow S^{1} * b=s,
$$

in which the first map retracts $[1 / 2,1]$ to $1 / 2$, the second collapses $S^{1} \times 1 / 2$ to $b$, and $S^{1} * b$, the cone over $S^{1}$ is identified with $s$ in the natural way. Now $q^{\prime}$ factors through $X_{p}$ so that we have the commutative diagram

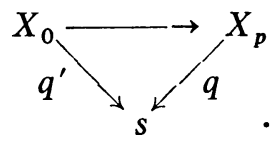

(I.1) $q^{-1}: C_{*}\left(s ; Z_{p}\right) \rightarrow C_{*}\left(X_{p} ; Z_{p}\right)$ is a chain map.

We must show that $\partial q^{-1}\left(\alpha \sigma^{i}\right)=q^{-1}\left(\partial \alpha \sigma^{i}\right), \sigma^{i} \in s, \alpha \in Z_{p}$. This is clear for $i=0,1$, as $q^{-1} \mid \partial s$ is $1-1$. For the only 2-simplex $\sigma^{2} \in s$ we get

$$
\partial q^{-1}\left(\alpha \cdot \sigma^{2}\right)=\alpha\left(\partial X_{p}\right)=\alpha\left(B_{p}+p \cdot C_{p}\right)=\alpha B_{p} \text {, as } p \alpha=0 .
$$

Finally, $q^{-1}\left(\partial \alpha \sigma^{2}\right)=\alpha \cdot q^{-1}\left(\partial \sigma^{2}\right)=\alpha \cdot B_{p}$.

Next suppose $p \neq p^{\prime}$. Then there are the maps $q: X_{p} \rightarrow s, q^{\prime}: X_{p^{\prime}} \rightarrow s$ and hence the product map $q \times q^{\prime}: X_{p} \times X_{p^{\prime}} \rightarrow s \times s$. Let $\hat{B}=\left(q \times q^{\prime}\right)^{-1}(\partial(s \times s))$ $=B_{p} \times X_{p^{\prime}} \cup X_{p} \times B_{p^{\prime}}$. Then

(I.2) There is a map $r: X_{p} \times X_{p^{\prime}} \rightarrow \partial(s \times s)=S^{3}$ such that $r\left|\hat{B}=q \times q^{\prime}\right| \hat{B}$.

Proof. We need only check the hypothesis of the Hopf extension theorem [4]. As $\left(X_{p} \times X_{p^{\prime}},\left(B_{p} \cup C_{p}\right) \times X_{p^{\prime}} \cup i X_{p} \times\left(B_{p^{\prime}} \cup C_{p^{\prime}}\right)\right)$ is a relative oriented manifold each homology class $v \in H_{4}\left(X_{p} \times X_{p^{\prime}}, \hat{B} ; S^{1}\right)$ has a representative of the form $\alpha \cdot X_{p} \times X_{p^{\prime}}, \alpha \in S^{1}$. Then $\partial v$ is represented by

$$
\alpha \partial\left(X_{p} \times X_{p^{\prime}}\right)=\alpha\left(\hat{B}+p C_{p} \times X_{p^{\prime}}+p^{\prime} \cdot X_{r} \times C_{n^{\prime}}\right)
$$


hence we must have $\alpha\left(p C_{p} \times X_{p^{\prime}}+p^{\prime} X_{p} \times C_{p^{\prime}}\right)=0$, and as $p \neq p^{\prime}, \alpha=0$. Hence $H_{4}\left(X_{p} \times X_{p^{\prime}}, \hat{B} ; S^{1}\right)=0$ and the map $r$ exists.

Let $K$ be a triangulated two-sphere and define $K_{i}$ inductively by $K_{i+1}=X_{p} \Delta K_{i}$. Define $\pi_{i}: K_{i+1} \rightarrow K_{i}$ by $q \Delta 1: X_{p} \Delta K_{i} \rightarrow K_{i}$. Let $\Phi_{p}$ be the inverse limit of the sequence $K_{1} \leftarrow{ }^{\pi_{1}} \quad K_{2} \leftarrow{ }^{\pi_{2}} K_{3} \leftarrow \cdots$.

(I.3) $\operatorname{dim} \Phi_{p^{\prime}}=2$, all primes $p$.

Proof. The inequality $\operatorname{dim} \Phi_{p} \leqq 2$ is easy. To check the other it suffices (by $\left.\mathrm{A}^{\prime}\right)$ to show that $\pi_{i^{*}}: H_{2}\left(K_{i+1} ; Z_{p}\right) \rightarrow H_{2}\left(K_{1} ; Z_{p}\right)$ is onto for $i=1,2, \cdots$, because $H_{2}\left(K_{1} ; Z_{p}\right)=Z_{p}$. But this follows from (I.1) and Corollary (2.4).

(I.4) $\operatorname{dim} \Phi_{p} \times \Phi_{p^{\prime}}=3$, for $p \neq p^{\prime}$.

Proof. The inequality $\geqq 3$ follows from general principles [2, p. 34]. To prove the other one, note that $\Phi_{p} \times \Phi_{p^{\prime}}$ is the inverse limit of the sequence

$$
K_{1} \times K_{1}^{\prime} \stackrel{\pi_{1} \times \pi_{1}^{\prime}}{\longleftarrow} K_{2} \times K_{2}^{\prime} \longleftarrow \cdots
$$

where $K_{i}, \pi_{i}$ are as above, and $K_{i}^{\prime}, \pi_{i}^{\prime}$ are the corresponding spaces and maps for $\Phi_{p^{\prime}}$. Regarding $K_{i} \times K_{i}^{\prime}$ as a CW-complex with cells $\sigma \times \sigma^{\prime}, \sigma \in K_{i}, \sigma^{\prime} \in K_{i}^{\prime}$, we can map each product of the form $\left(X_{p}, \sigma\right) \times\left(X_{p}, \sigma^{\prime}\right)$ into $\partial\left(\sigma \times \sigma^{\prime}\right)$ using the map $r$ of (I.2). These are consistent throughout $K_{i+1} \times K_{i+1}^{\prime}$ because on the intersection of two such products, $r$ agrees with $\pi_{i}$ which is consistent. This yields a map $\psi: K_{i+1} \times K_{i+1}^{\prime} \rightarrow\left(3-\right.$ skeleton of $\left.K_{i} \times K_{i}^{\prime}\right)$ which is a cellular approximation to $\pi_{i} \times \pi_{i}^{\prime}$ so that by $\left(\mathrm{A}^{\prime}\right), \operatorname{dim} \Phi_{p} \times \Phi_{p^{\prime}} \leqq 3$.

II. (BoltYANSKII [2]). There is a compact metric space $X$ of dimension 2 such that $\operatorname{dim} X \times X=3$.

Proof. Fix a prime $p$ and let $n_{i}=p^{2^{i}}, i=1,2,3, \cdots$. Let $X_{0}=S^{1} \times[0,1]$ and let $X_{i}$ be the space formed from $X_{0}$ by identifying $(\theta, 0) \sim\left(\theta+2 \pi / n_{i}, 0\right)$ and $(\theta, 1) \sim\left(\theta+2 \pi / n_{i+1}, 1\right)$, for all $\theta \in S^{1}$. Let $B_{i} \subset X_{i}$ correspond to $S^{1} \times 0$ and $C_{i}$ to $S^{1} \times 1$. Then $\left(X_{i}, B_{i} \cup C_{i}\right)$ is a relative, orientable manifold and $\partial X_{i}=n_{i} B_{i}$ $+n_{i+1} C_{i}$. Define $q^{\prime}: X_{0} \rightarrow s$ by

$$
q^{\prime}: S^{1} \times[0,1] \underset{n_{i}}{\longrightarrow} S^{1} \times[0,1] \rightarrow S^{1} * b=s
$$

in which the first map sends $S^{1} \rightarrow S^{1}$ by $\theta \rightarrow n_{i} \theta$ for all $\theta \in S^{1}$, and the second is as in (I) above.

Then $q^{\prime}$ factors through $X_{i}$ defining $q_{i}$ :

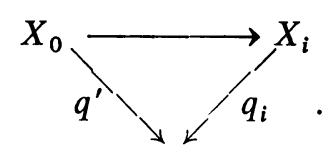

$S$

Below we consider $Z_{n_{i}} \subset Z_{n_{i+1}} \subset S^{1}$, the reals mod 1, and choose generators $\alpha_{i} \in$ $Z_{n_{i}}$, such that $n_{i} \alpha_{i+1}=\alpha_{i}$.

(II.1) There is a chain map $\gamma_{i}$ such that the following diagram 


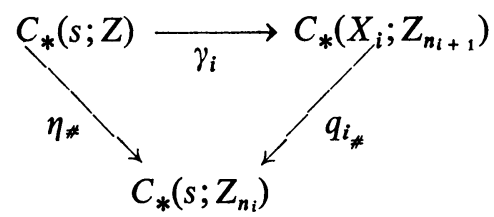

is commutative, in which $\eta_{\#}$ is induced by the natural map $\eta: Z \rightarrow Z_{n_{i}}$.

Proof. For a $j$-simplex $\sigma^{j} \in s, n \in Z$, define

$$
\gamma_{i}\left(n \cdot \sigma^{j}\right)= \begin{cases}n \alpha_{i} q_{i}^{-1}\left(\sigma^{j}\right), & j=0,1 ; \\ n \alpha_{i+1} q_{i}^{-1}\left(\sigma^{j}\right), & j=2 .\end{cases}
$$

The relation $q_{i \neq *} \gamma_{i}=\eta_{i}$ follows from the fact that $q_{i}$ is $n_{i}$-to-1 on $X_{i}-B_{i} \cup C_{i}$, 1-1 on $B_{i}$ and $n_{i+1}$-to-1 on $C_{i}$. As in (I) we need only check $\partial \gamma_{i}\left(n \cdot \sigma^{2}\right)=\gamma_{i}\left(n \partial \sigma^{2}\right)$ for the unique 1-simplex $\sigma^{2} \in s$. But $\partial \gamma_{t}\left(n \sigma^{2}\right)=n \alpha_{i+1} \partial X_{i}=n \alpha_{i+1}\left(n_{i} B_{i}+n_{i+1} C_{i}\right)$ $=n \alpha_{i} B_{i}$ and $\gamma_{i}\left(\partial n \cdot \sigma^{2}\right)=n \alpha_{i} q_{i}^{-1}\left(\partial \sigma^{2}\right)=n \alpha_{i} B_{i}$.

Now let $\hat{B}_{i}=B_{i} \times X_{i} \cup X_{i} \times B_{i}=\left(q_{i} \times q_{i}\right)^{-1}(\partial(s \times s))$. Then

(II.2) There is a map $r_{i}: X_{i} \times X_{i} \rightarrow \partial(s \times s) \approx S^{3}$ such that $r_{i}\left|\hat{B}_{i}=\left(q_{i} \times q_{i}\right)\right| \hat{B}_{i}$.

Proof. As before, every homology class $v \in H_{4}\left(X_{i} \times X_{i}, \hat{B}_{i} ; S^{1}\right)$ has a representative of the form $z=\alpha \cdot X_{i} \times X_{i}, \alpha \in S^{1}$. Then $\partial z=\partial\left(\alpha \cdot X_{i} \times X_{i}\right)$ $=\alpha\left(n_{i} B_{i}+n_{i+1} C_{i}\right) \times X_{i}+\alpha \cdot X_{i} \times\left(n_{i} B_{i}+n_{i+1} C_{i}\right)$. Hence $\alpha n_{i+1} C_{i} \times X_{i}$ $+\alpha \cdot n_{i+1} X_{i} \times C_{i}=0$ and $n_{i+1} \alpha=0$ (and thus $H_{4}\left(X_{i} \times X_{i}, \hat{B}_{i} ; S^{\prime}\right)=Z_{n_{i+1}}$ ). Then $q_{i \neq} \partial z=q_{i \neq}\left(\alpha n_{i} B_{i} \times X_{i}+\alpha n_{i} X_{i} \times B_{i}\right)=\alpha n_{i}(\partial s) \times\left(n_{i} s\right)+\alpha n_{i}\left(n_{i} s\right) \times(\partial s)$ $=\alpha n_{i+1} \cdot \partial(s \times s)=0$, and $r_{\imath}$ exists by the Hopf extension theorem.

As in (I) define $X$ as the inverse limit of

$$
K_{1} \stackrel{\pi_{1}}{\longleftarrow} K_{2} \stackrel{\pi_{2}}{\longleftarrow} K_{3} \longleftarrow \cdots
$$

where $K_{1}=S^{2}$ and $K_{i+1}=X_{i+1} \Delta K_{i} \cdot \pi_{i}: K_{i+1} \rightarrow K_{i}$ is given by $q_{i+1} \Delta 1: X_{i+1} \Delta K_{i}$ $\rightarrow K_{i}$.

(II.3) $\operatorname{dim} X \times X=3$ follows just as in I from (II.2).

(II.4) $\operatorname{dim} X=2$.

Proof. That $\operatorname{dim} X \geqq 2$ follows just as in (I), but the other inequality is a bit different because of the change of coefficients in (III.1). The claim is that $H_{2}\left(X ; S^{1}\right) \neq 0$ and this will follow from

$$
\text { (4a) }\left\{\begin{array}{l}
\pi_{1 *}: H_{2}\left(K_{2} ; S^{1}\right) \rightarrow H_{2}\left(K_{1} S^{1}\right) \text { is nontrivial, } \\
\pi_{i_{*}}: H_{2}\left(K_{i+1} ; S^{1}\right) \rightarrow H_{2}\left(K_{i} ; S^{1}\right) \text { is onto, } i=2,3, \cdots .
\end{array}\right.
$$

We need only consider the cycle groups $Z_{2}\left(K_{i} ; S^{1}\right)$ as we are in the top dimension. But as in the proof of (II.2), $Z_{2}\left(K_{i} ; S^{1}\right)=Z_{2}\left(K_{i} ; Z_{n i+1}\right)$. Now it follows from (II.1) and Corollary (2.4) that $\pi_{i_{\text {t\# }}}: C_{2}\left(K_{i+1} ; Z_{n_{i+2}}\right) \rightarrow Z_{2}\left(K_{i} ; Z_{n_{i+1}}\right)$ is onto. But any 2-chain which maps onto a cycle under $\pi_{i \neq}$ must be a cycle, as $\pi_{i}$ | $\pi_{1}^{-1}\left(1\right.$-skeleton of $\left.K_{i}\right)$ is a homeomorphism. 
III. (KolmogorofF [5]). There is a compact 1-dimensional space $Y$ and a compact abelian group $A$ acting freely on $Y$ such that $\operatorname{dim} Y / A=2$.

Proof. This is based on example $I$ and we let the notation of (I) stand. Let $\gamma_{t}: S^{1} \rightarrow S^{1}$ be the identity for $0 \leqq t \leqq 1 / 2$, and a rotation for $1 / 2 \leqq t \leqq 1, \gamma_{1}$ being a rotation of period $p$. Let $Y_{0}=S^{1} \times[0,1] \times Z_{p}$ and define $f_{0}, g_{0}: Y_{0} \rightarrow Y_{0}$ by

$$
\begin{aligned}
& f_{0}(x, t, \alpha)=(x, t, \alpha+\omega), \quad \omega \text { a generator of } Z_{p}, \\
& g_{0}(x, t, \alpha)= \begin{cases}\left(\gamma_{t} x, t, \alpha+\omega\right), & \alpha \neq 0, \\
\left(\gamma_{t}^{-p+1} x, t, \omega\right), & \alpha=0 .\end{cases}
\end{aligned}
$$

Let $A=S^{1} \times 1 \times Z_{p}$. Then on $A, g_{0}(x, t, \alpha)=\left(\gamma_{t} x, t, \alpha+\omega\right)$ so that $f_{0} \mid A$ and $g_{0} \mid A$ commute.

Let $Y_{p}=Y_{0} / g_{0} \mid A$. Then $f_{0}, g_{0}$ induce maps $f_{p}, g_{p}: Y_{p} \rightarrow Y_{p}$ both of period $p$ and $f_{p}$ has no fixed point. Define $q_{1}^{\prime}: Y_{0} \rightarrow s$ by

$$
q_{1}^{\prime}: Y_{0} \rightarrow Y_{0} / g_{0}=X_{0} \rightarrow s
$$

in which the first map is the orbit map and the second is $q^{\prime}$, defined in (I). Then $q_{1}^{\prime}$ collapses all orbits of $g_{0}$.

Furthermore $q_{1}^{\prime}$ collapses all orbits of $f_{0}$. For this there are two cases: $0 \leqq t \leqq 1 / 2$ for which $g_{0}(x, t, \alpha)=f_{0}(x, t, \alpha)$, and the $f_{0}$-orbits $=g_{0}$-orbits; for $1 / 2 \leqq t \leqq 1, q_{1}^{\prime}(x, t, \alpha)=b$ and $q_{1}^{\prime} f_{0}(x, t, \alpha)=q_{1}^{\prime}(x, t, \alpha+\omega)=b$ as well.

Hence there are the induced maps

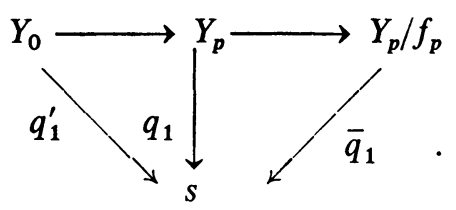

(III.1) $Y_{p} / f_{p} \approx X_{p}$ and identifying these $\bar{q}_{1}=q: X_{p} \rightarrow s$.

Proof. First $Y_{p} / f_{p}=\left(Y_{0} / g_{0} \mid A\right) / f_{p}=\left(Y_{0} / f_{p}\right) / g_{p} \mid A^{\prime}=X_{0} /(\theta, 1) \sim(\theta+2 \pi / p, 1)$ $=X_{p}$. The second statement follows because $q_{1}^{\prime}$ was defined in terms of $q^{\prime}$.

(III.2) There is a map $r_{1}: Y_{p} \rightarrow \partial s$ such that

$$
r_{1} q^{-1}(\bar{\sigma}) \subset \bar{\sigma} \text { for all } \sigma \in ; .
$$

Proof. Define $r_{1}^{\prime}: Y_{0} \rightarrow \partial s$ by $r_{1}^{\prime}: Y_{0} \rightarrow Y_{0} / g_{0}=S^{1} \times[0,1] \rightarrow S^{1}=\partial s$. Then as $r_{1}^{\prime}$ collapses all orbits of $g_{0}, r_{1}^{\prime}$ factor through $Y_{p}$ :

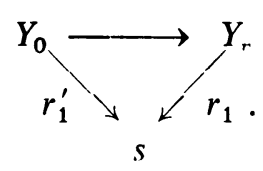


Note that $r_{1} q^{-1}(\bar{\sigma}) \subset \bar{\sigma}$, for $\sigma=\sigma^{0}, \sigma^{1} \in s$, as here $r_{1}=q_{1}$. For $\sigma=\sigma^{2}$ this condition is trivial.

Let $L_{1}=S^{2}$ and define $L_{i}$ inductively by $L_{i+1}=Y_{p} \Delta L_{i}$. Define $\pi_{i 1}: L_{i+1} \rightarrow L_{i}$ by $q \Delta 1: Y_{p} \Delta L_{i} \rightarrow L_{i}$, and $Y=$ inverse limit of $\left(L_{i}, \pi_{i 1}\right)$. Let $F_{i 0}: L_{i} \rightarrow L_{i}$ be the identity and define $F_{i 1}, \cdots, F_{i i}: L_{i} \rightarrow L_{i}$ inductively by

$$
F_{i+1, j}=f_{p} \Delta F_{i, j-1}, \quad j=1,2, \cdots, i+1 .
$$

Note that $F_{i+1, j}$ has no fixed point because $f_{p}$ has none.

Then $\left\{F_{i j}\right\}_{j=1}^{i}$ generates a free action of $Z_{p}+\cdots+Z_{p}$ ( $i$ summands) upon $L_{i}$, and

(III.3) $\pi_{i 1}: L_{i+1} \rightarrow L_{i}$ is equivariant. That is

For

$$
\pi_{i 1} F_{i+1, j}=F_{i, j-1} \pi_{i 1}, \quad j=1,2, \cdots, i .
$$

$$
\begin{aligned}
\pi_{i 1} F_{i+1, j} & =\left(q_{1} \Delta 1\right)\left(f_{p} \Delta F_{i, j-1}\right)=q_{1} f_{p} \Delta F_{i, j-1} \\
& =q_{1} \Delta F_{i, j-1}=\left(1 \Delta F_{i j-1}\right)\left(q_{1} \Delta 1\right)=F_{i, j-1} \pi_{i 1} .
\end{aligned}
$$

Let $[f, g, \cdots]$ denote the group generated by $f, g, \cdots$. Then define the abelian group $A$ as the inverse limit

$$
\left[F_{10}\right] \stackrel{\pi_{11}}{\longleftarrow}\left[F_{2 j}\right]_{j=1}^{1} \stackrel{\pi_{21}}{\longleftarrow}\left[F_{3 j}\right]_{j=1}^{2} \stackrel{\pi_{31}}{\longleftarrow} \cdots .
$$

Then $A$ acts on $Y$, coordinatewise and thus freely. This uses the equivariance, (III.3).

(III.4) $\operatorname{dim} Y=1$.

Proof. We prove only $\operatorname{dim} Y \leqq 1$, and hence need only check the hypothesis of $\left(A^{\prime}\right)$. There is the map

$$
1 \Delta 1: Y_{p} \Delta_{q_{1}} L_{i} \rightarrow Y_{p} \Delta_{r_{1}} L_{i}
$$

because $r_{1} q^{-1}(\bar{\sigma})=r q^{-1}(\bar{\sigma}) \subset \bar{\sigma}$ for all $\sigma \in s$. Now define $\psi$ by

$$
\psi: Y_{p} \Delta_{q} L_{i} \stackrel{1 \Delta 1}{\longrightarrow} Y_{p} \Delta_{r_{1}} L_{i} \stackrel{r_{1} \Delta 1}{\longrightarrow}(\partial s) \Delta L_{i}=1 \text {-skeleton of } L_{i} .
$$

This is clearly a simplicial approximation to $\pi_{i 1}$ and $\left(\mathrm{A}^{\prime}\right)$ is verified.

(III.5) $Y / A=\Phi_{p}$ so that $\operatorname{dim} Y / A=2$.

Proof. By induction; we note that $L_{1} / F_{10}=S^{2} / 1=K_{1}$. Now

$$
\begin{aligned}
L_{i+1} /\left[F_{i+1,1}, \cdots, F_{i+1, i+1}\right] & =Y_{p} / f_{p} \Delta\left(L_{i} /\left[F_{i, 0}, \cdots, F_{i, i}\right]\right) \\
& =\left(Y_{p} / f_{p}\right) \Delta\left(L_{i} /\left[F_{i, 1}, \cdots, F_{i, i}\right]\right)=\left(Y_{p} f_{p}\right) \Delta K_{i}
\end{aligned}
$$

by the induction hypothesis. But by (III.1) this last is $X_{p} \Delta K_{i}=K_{i+1}$. Hence $Y / A$ is the inverse limit of

$$
K_{0} \longleftarrow K_{1} \longleftarrow \cdots
$$

in which the connecting maps are $\bar{q}_{1} \Delta 1=q \Delta 1: X_{p} \Delta K_{i} \rightarrow K_{i}$. But this is precisely the definition of $\Phi_{p}$. 
REMARKS. (1) Kolmogoroff, writing in 1937, did not express his ideas in terms of transformation groups. His concern was to construct an open map which raised dimension and the map he gives is precisely the orbit map of an action of a group $A, Y^{\prime} \rightarrow Y^{\prime} / A=\Phi_{p}$, for $p=2$. A notable difference is that in his example the (implicit) action of $A$ is far from free.

$Y^{\prime}$ can be described (for any $p$ ) just as $Y$, except that in place of $Y_{p}$ one uses $Y_{p}^{\prime}$ defined by $Y_{p}=Y_{0} / g_{0} \mid A^{\prime}$, where $A^{\prime}=S^{1} \times\{0,1\} \times Z_{p}$. On $Y^{\prime}$ one can equally well define the action of the $p$-adic group $A_{p}$ (given as the inverse limit of the sequence $\left.Z_{p} \leftarrow Z_{p^{2}} \leftarrow Z_{p^{3}} \leftarrow \cdots\right)$ so that $Y^{\prime} / A_{p}=\Phi_{p}$. This version of Kolmogoroff's is then closer to that given in $[7 ; 8]$, in which $A_{p}$ acts (not freely) on a 2-dimensional space $X$ and $\operatorname{dim} X / A_{p}=4$.

Finally, one can define a 1-dimensional space $Y^{\prime \prime}$ and a free action of $A_{p}$ on $Y^{\prime \prime}$ such that $\operatorname{dim} Y^{\prime \prime} / A_{p}=2$. This differs from the example above in two ways. First, instead of $Y_{p}$ one uses $Y_{p}^{\prime \prime}=Y_{0}^{\prime \prime} / g_{0}{ }^{\prime \prime} \mid A^{\prime \prime}$, where $Y_{0}^{\prime \prime}=D_{p} \times Z_{p} . D_{p}$ is a 2-dimensional disk from which have been deleted $p$ open disks with boundaries $A_{0}, \cdots, A_{p-1} ; A^{\prime \prime}=\bigcup A_{i}$. The map $g_{0}^{\prime \prime}: Y_{0}^{\prime \prime} \rightarrow Y_{0}^{\prime \prime}$ takes $A_{a} \times b \rightarrow A_{a+\omega} \times(b+\omega)$, whereas $f_{0}^{\prime \prime}: A_{a} \times b \rightarrow A_{a} \times(b+\omega)$, for $a, b \in Z_{p}$. The crucial difference is that the co-index [3] (or $B$-index, [9]) of $\left(Y_{p}^{\prime \prime}, f_{p}{ }^{\prime \prime}\right)$ is 1 ; that of $\left(Y_{p}, f_{p}\right)$ is 2 .

Next, instead of defining $L_{i}^{\prime \prime}=Y_{p}^{\prime \prime} \Delta L_{i}^{\prime \prime}$, one uses a more complicated inductive procedure, just as in [8], making use of the fact that co-index $\left(Y_{p}^{\prime \prime}, f_{p}^{\prime \prime}\right)=1$. Generally speaking, the higher the co-index, the "deeper" the example. This topic will be pursued in a future paper.

\section{BIBLIOGRAPHY}

1. R. D. Anderson, Zero dimensional compact groups of homeomorphisms, Pacific J. Math. 7 (1957), 799.

2. V. Boltyanskii, An example of a two-dimensional compactum whose topoligical square is three-dimensional, Amer. Math. Soc. Transl. No. 48, (1951), 3-6; [Dokl. Akad. Nauk SSSR 67 (1949), 597-599].

3. P. E. Conner and E. E. Floyd, Fixed point free involutions and equivariant maps, Bull. Amer. Math. Soc. 66 (1960), 420.

4. Hurewicz and Wallman, Dimension theory, Princeton Univ. Press, Princeton, N. J., revised edition, 1948.

5. A. Kolmogoroff, Über offene Abbildungen, Ann. of Math. (2) 38 (1937), 36-38.

6. L. Pontrjagin, Sur un hypothèse fondamental de la théorie de la dimension, C. R. Acad. Sci. Paris 130 (1930), 1405-1407.

7. Frank Raymond and R. F. Williams, Examples of p-adic transformation groups, Bull. Amer. Math. Soc. 66 (1960), 392-394.

8. ——, Examples of p-adic transformation groups, (to appear).

9. C. T. Yang, Continuous functions from spheres to Euclidian to spaces, Ann. of Math. (2) 62 (1955), 284-292.

THE UNIVERSITY OF CHICAGo,

Chicago, Illinois 